\title{
Pharmacogenomic testing to prevent aminoglycoside-induced hearing loss in cystic fibrosis patients: potential impact on clinical, patient, and economic outcomes
}

David L. Veenstra, PharmD, $P h D^{1,2}$, Julie Harris, $M P H^{1}$, Ronald L. Gibson, $M D, P h D^{3,4}$, Margaret Rosenfeld, $M D, M P H^{3,4}$, Wylie Burke, $M D, P h D^{1,5}$, and Carolyn Watts, $P h D^{1,6}$

\begin{abstract}
Background: Aminoglycosides are commonly used in cystic fibrosis patients to treat Pseudomonas aeruginosa respiratory infections. Aminoglycoside-induced hearing loss may occur in $1 \%-15 \%$ of patients with cystic fibrosis, ranging from mild to severe. Recently, a genetic test to identify patients with a mitochondrial mutation (A1555G) that may predispose patients to this adverse event has become available. Although the A1555G variant is very rare, it seems to confer a high risk of severe hearing loss in patients exposed to aminoglycosides. Objective: The objective was to evaluate the potential clinical, patient, and economic outcomes associated with the use of A1555G testing in a cystic fibrosis population, and explore data gaps and uncertainty in its clinical implementation. Methods: We developed a decision-analytic model to evaluate a hypothetical cohort of patients with cystic fibrosis from a societal perspective. Clinical and economic data were derived primarily from a critical literature review. The incidence of aminoglycoside-induced severe hearing loss, quality-adjusted life-years, and total health care costs were evaluated. Sensitivity analyses were conducted to evaluate uncertainty in our results. Results: In the base-case analysis, A1555G testing decreased the risk of severe aminoglycoside-induced hearing loss by $0.12 \%$ in the cystic fibrosis population. The discounted incremental cost per quality-adjusted life-years gained was $\$ 79,300$, but varied widely from $\$ 33,000$ to testing being dominated by the no testing strategy (higher costs and lower quality-adjusted life-years with testing) in sensitivity analyses. If avoidance of aminoglycosides in patients testing positive leads to an absolute increase in the lifetime risk of death from Pseudomonas infection of $0.8 \%$ or greater, A1555G testing would lead to a decrease in quality-adjusted life-years. Conclusions: The results of our analysis suggest that there are significant data gaps and uncertainty in the outcomes with A1555G testing, but it is not likely cost-effective, and could lead to worse patient outcomes due to avoidance of first-line therapy in the $>95 \%$ of patients who are false-positives. Additional research is needed before pharmacogenetic testing for the A1555G mitochondrial mutation can be recommended, even in a population with a high likelihood of exposure to aminoglycosides. Genet Med 2007:9(10):695-704.
\end{abstract}

Key Words: pharmacogenomics, mitochondrial mutation, hearing loss, adverse drug event, cost-effectiveness

Aminoglycoside antibiotics are commonly used to treat serious Gram-negative bacterial infections such as Pseudomonas aeruginosa and multidrug-resistant Mycobacterium tuberculo-

From the ${ }^{1}$ Institute for Public Health Genetics, and Departments of ${ }^{2}$ Pharmacy and ${ }^{3}$ Pediat rics, University of Washington, Seattle, Washington; ${ }^{4}$ Seattle Children's Hospital and Regional Medical Center, Division of Pulmonary Medicine, Seattle, Washington; Departments of ${ }^{5}$ Medical History and Ethics, and ${ }^{6}$ Health Services, University of Washington, Seattle, Washington.

David L. Veenstra, PharmD, PhD, Department of Pharmacy, Box 357630, University of Washington, Seattle, WA 98195. E-mail: veenstra@u.washington.edu.

Disclosure: The authors declare no conflict of interest.

Submitted for publication April 13, 2007.

Accepted July 2, 2007.

DOI: 10.1097/GIM.0b013e318156dd07 sis. ${ }^{1}$ Although this class of drugs is highly effective, it has also been shown to have side effects resulting in renal and ototoxicity. ${ }^{2-5}$ Specifically, a recent article estimated that approximately $7 \%$ of all patients exposed to aminoglycosides experience some form of cochleotoxicity, although there is significant uncertainty associated with the risk, with a range of reported values from $0 \%$ to $16 \%{ }^{6}$ Aminoglycoside-induced hearing loss is classified as sensorineural hearing loss and mainly affects hearing loss at high frequencies. ${ }^{7} \mathrm{Al}-$ though there are not common definitions of hearing loss across studies, it seems that a majority of individuals experience subclinical to moderate hearing loss, with an estimated $1-4 \%$ of all patients experiencing more severe hearing loss. ${ }^{8,9}$ 
There are several variants in the mitochondrial $12 \mathrm{~S}$ ribosomal rRNA gene that are believed to be associated with aminoglycoside-induced hearing loss, including variants in positions 961, 1494, and 1555. ${ }^{10,11}$ The most common of these, A1555G, has been reported to have an association with bilateral sensorineural hearing loss across a number of populations worldwide. ${ }^{10,12-18}$ The mutation was first reported in a large Arab-Israeli pedigree with known matrilineal transmission of hearing loss. ${ }^{19}$ Since then the mutation has been reported in deaf populations worldwide with estimates ranging from approximately $0.7 \%$ in a cohort of German patients ${ }^{20}$ to approximately $20 \%$ in a cohort of Spanish patients with a family history of hearing loss. ${ }^{21}$ Few studies have examined the prevalence of the mutation in the general population. In one US study, Tang et al. collected a random sample of 1173 blood spots from newborns in the state of Texas. A single mutation was identified, resulting in an estimated population prevalence of $0.00086\left(8.6\right.$ per 10,000). ${ }^{14}$

Few estimates of the association of this mutation with hearing loss in individuals exposed versus not exposed to aminoglycosides are available. Those studies reporting on extended families exhibiting matrilineal transmission of hearing loss found that all identified individuals who were exposed to an aminoglycoside experienced some form of hearing loss. Thus, a recent review on the web site GeneTests estimates the penetrance of this mutation in individuals exposed to aminoglycosides to be approximately $100 \% .{ }^{22}$

Although there is a paucity of evidence documenting the association between the $\mathrm{A} 1555 \mathrm{G}$ variant and aminoglycosideinduced hearing loss, a clinical genetic test is currently available. ${ }^{23}$ Because 7-14 days are required to receive test results, test results must be known prior to onset of a Gram-negative bacterial infection to achieve clinical utility. The intended use thus seems to be as a screening tool, particularly for patients likely to receive aminoglycoside therapy.

Patients with cystic fibrosis (CF) may be ideal candidates for the $\mathrm{A} 1555 \mathrm{G}$ test. CF is the most common life-shortening genetic disease in the Caucasian population with an estimated 30,000 diagnosed patients in the US and $\sim 60,000$ patients worldwide. CF is a genetic disorder characterized by progressive obstructive lung disease, chronic airway infection, repeated pulmonary exacerbations requiring intensification of therapy, and a reduced life expectancy. Currently, the median predicted survival is estimated to be 36.5 years. ${ }^{24}$

Patients with CF have chronic airway infection from early childhood, with an initial predominance of Staphylococcus aureus in the preschool population, and then an increasing prevalence of $P$. aeruginosa in older children and adults. ${ }^{25}$ The Cystic Fibrosis Foundation Registry reports that approximately $80 \%$ of adult US CF patients have chronic Pseudomonas infection. ${ }^{26}$ Patients with CF have a recurrent need for intravenous antibiotics to treat pulmonary exacerbations. In individuals infected with $P$. aeruginosa, the antibiotic regimen of choice is the combination of an aminoglycoside and a $\beta$-lactam. In addition, the majority of US school-aged children and adults with CF who are chronically colonized with Pseudomonas receive maintenance therapy with alternate-month inhaled tobramycin to improve lung function and decrease the rate of pulmonary exacerbations, although the inhaled form of tobramycin has not been associated with hearing loss. ${ }^{25}$

The primary objectives of this study was to estimate the potential incremental clinical and economic outcomes associated with screening for the $\mathrm{A} 1555 \mathrm{G}$ variant in CF patients who have not yet received aminoglycoside therapy. The results of this analysis will help guide decisions about clinical use of and reimbursement for the A1555G test, and identify areas for further research.

\section{MATERIALS AND METHODS}

\section{Overview}

We developed a decision analytic model to evaluate the incremental clinical, patient, and economic outcomes associated with the use of the A1555G test in a hypothetical cohort of patients with CF from the societal perspective. Inputs for the model were identified from a critical review of the literature and data from the US Cystic Fibrosis Foundation National Patient Registry, in collaboration with CF center staff at Children's Hospital and Regional Medical Center, Seattle, WA. The incremental cost-effectiveness ratio (ICER) was the main outcome of the study, reported as the incremental cost to produce one additional year with perfect quality of life. We also calculated the differences in the incidence of severe aminoglycosideinduced hearing loss, quality-adjusted life-years (QALYs), and total health care costs. Analyses were performed using TreeAgePro 2005 software ${ }^{27}$ (Willamstown, MA) and Microsoft Excel $^{\circledR}$ (Redmond, WA). Costs and outcomes were discounted at a standard 3\% rate.

Data used to inform our model were gathered from a review of the available literature on the A1555G variant and its association with hearing loss. MEDLINE searches spanning the years 1966-2005 were conducted using the search terms ototoxicity, A1555G, hearing loss, mitochondrial, aminoglycosides, and genetics. Bibliographies of all manuscripts that were included to inform our model were also reviewed, and additional studies were identified for inclusion. Because of the paucity of research on this topic, we included all empirical studies of the A1555G variant, including family-based studies, pedigree studies, and studies conducted in hearing impaired and nonhearing impaired populations. We did not include anecdotal or case-study reports in the evidence used to inform our model.

\section{Hypothetical patient population}

Guidelines for the treatment of moderate to severe pulmonary exacerbations in CF patients infected with $P$. aeruginosa recommend parenteral administration of two antibiotics over $7-21$ days. ${ }^{28}$ There are three classes of antibiotics available, including $\beta$-lactams, aminoglycosides, and quinolones. Aminoglycosides, typically tobramycin, in conjunction with a $\beta$-lactam are the preferred treatment, ${ }^{28}$ likely because there is some evidence indicating that $20 \%$ of Pseudomonas infections 
are resistant to quinolones (e.g., ciprofloxicin), whereas $4 \%$ are resistant to tobramycin, ${ }^{29}$ and because of the synergistic bactericidal effect of the combination of an aminoglycoside and a $\beta$-lactam. In this analysis, we assumed that patients testing positive for the mutation would receive a $\beta$-lactam and a quinolone (intravenous ciprofloxicin).

\section{Model structure}

We developed a decision model to compare testing for the A1555G mutation with no testing (standard care) in patients with CF (Fig. 1). The probability of patients receiving an intravenous aminoglycoside was based on data collected from US Cystic Fibrosis Foundation National Patient Registry. ${ }^{30}$ Genotypes of patients in the hypothetical cohort were based on the prevalence of the mutation in the general population, and were held constant for both strategies. In patients receiving the genetic test, those with the mutation were treated with intravenous ciprofloxacin and ceftazidime whereas those testing negative were treated with tobramycin and ceftazidime. Patients in the standard care group received tobramycin and ceftazidime.

\section{Key assumptions}

Given the relative paucity of data on the incidence, severity, and timing of aminoglycoside-induced hearing loss, as noted above, and the few data available on hearing loss and the A1555G mutation, several assumptions were required to estimate the potential clinical and economic utility of the test. The following assumptions were employed:

- We estimated that $6 \%$ of mutation-negative patients experience mild or moderate aminoglycoside-induced hearing loss, but varied this estimate from $3 \%$ to $9 \%$ given the wide range of values reported in the literature. ${ }^{6}$ Similarly, we estimated $1.2 \%$ of all patients experience severe aminoglycoside-induced hearing loss, with a range from $0.6-1.8 \% .^{8}$

- Although exposure to aminoglycosides can happen at any point over the lifetime of a person with CF, we estimated the average age at first exposure to intravenous tobramycin of approximately 12 years, given that the age at which $50 \%$ of patients have Pseudomonas colonization is 10 years, and $30 \%$ of patients below age $13,40 \%$ of patients $14-17$ years, and $50 \%$ of patients $18-30$ were hospitalized at least once in 2005 related to a Pseudomonas infection. ${ }^{26}$

- As repeated exposure to aminoglycosides has been shown to increase the risk of hearing loss over time, we assumed hearing loss occurred, on average, at the midpoint of life expectancy (17 years), 5 years after first exposure to aminoglycoside. $6,24,30,31$

- Individuals who developed either early or late-onset severe or profound hearing loss received a cochlear implant. Several studies suggest that the mechanism by which aminoglycoside-induced cochleotoxicity occurs may preserve cochlear nerve function. ${ }^{32-34}$ Therefore, individuals with this specific type of pathology are believed to be good candidates for cochlear implantation.
- We assumed that approximately $35 \%$ of individuals in the mild or moderate hearing loss would have a hearing aid. This assumption was based on data from the Hearing Review 2006 Dispenser Survey indicating that approximately $16 \%$ of sampled individuals with mild hearing loss and $53 \%$ of those with moderate hearing loss have hearing aids ( $35 \%$ is the average of these two). ${ }^{35}$

- We did not evaluate the risk of aminoglycoside-induced hearing loss due to inhaled tobramycin, as peak drug serum levels are generally $<2 \mu \mathrm{g} / \mathrm{mL}$ compared with peak levels of $10 \mu \mathrm{g} / \mathrm{mL}$ IV thrice daily or $>40 \mu \mathrm{g} / \mathrm{mL}$ using once daily intravenous tobramycin, and in Phase III trials of inhaled tobramycin there was no increased high frequency hearing loss in the treatment arm compared with placebo arm. ${ }^{25}$

- In the base-case we assumed that there was no difference in mortality between patients receiving quinolones + $\beta$-lactam versus those receiving aminoglycoside $+\beta$-lactam. This assumption was based on evidence from one randomized clinical trial suggesting equivalent efficacy of quinolones to aminoglycosides at eradicating Gram-negative bacterial infections. ${ }^{36}$ Because there is evidence of higher rates of quinolone-resistant Pseudomonas infections compared with tobramycin-resistant infections in CF patients, ${ }^{29}$ we assessed the potential negative impacts of this drug switch. We estimated a lifetime attributable mortality from Pseudomonas infection in CF of $28 \%$ based on an increase in the relative risk of death of $50 \%$ associated with Pseudomonas exacerbation, a life expectancy of approximately 35 years, and a 50\% probability of a Psuedomonas exacerbation. ${ }^{26,37,38}$ We then estimated a $10 \%$ relative increase in lifetime mortality with ciprofloxacin versus tobramycin to obtain a higher absolute risk of death of $2.8 \%$ attributable to lifetime use of second-line therapy.

Four possible outcomes were thus assessed with respect to hearing loss in each strategy. The first was "aminoglycosideinduced severe hearing loss," defined as severe hearing loss resulting from exposure and persisting for 17 years (remainder of life-expectancy). The second was "aminoglycoside-induced mild hearing loss," defined as mild hearing loss resulting from exposure and persisting for 17 years. The third was "severe late-onset hearing loss" defined as severe hearing loss with an assumed age at onset of 27 years and persisting for the remainder of one's life (i.e., 7 years). This definition of late-onset hearing loss was determined based on a study by Matsunaga et al. reporting a 10-year difference between early and late hearing loss onset. ${ }^{39}$ The fourth outcome was no hearing loss.

\section{Clinical probabilities}

The literature review revealed a paucity of information about the mutation prevalence, and only a few studies reported an association with hearing loss in the absence of aminoglycoside exposure. ${ }^{15,39}$ In an investigation of Spanish families with sensorineural deafness, Estivill and colleagues reported that 


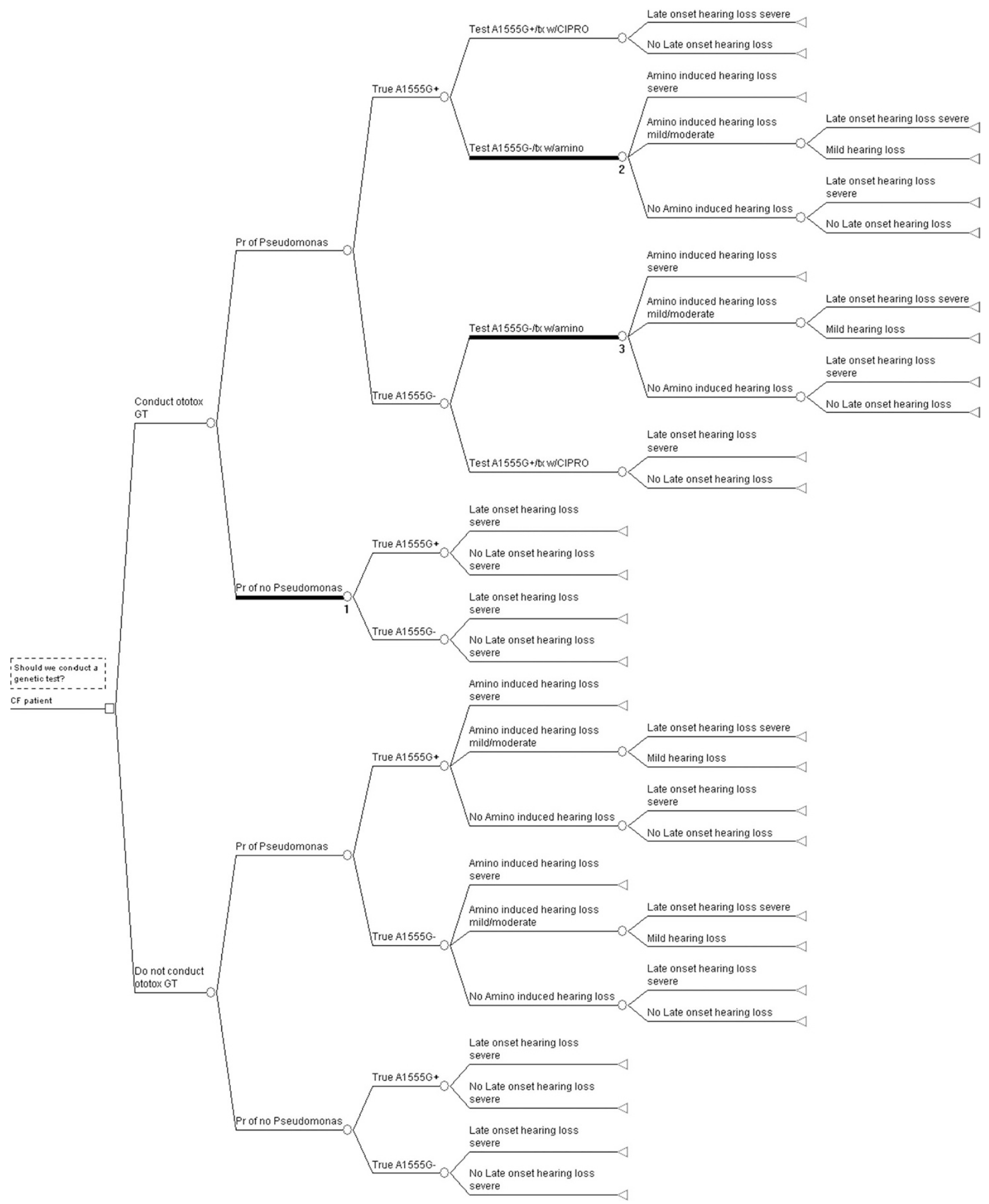

Fig. 1. Structure of decision tree representing testing and treatment algorithm. AG, aminoglycoside.

$39.9 \%$ of those with the mutation who were not exposed to aminoglycosides developed hearing loss. ${ }^{40}$ In another study Matsunaga et al. found that age at onset differed between probands who were exposed and unexposed by approxi- mately 10 years, with exposed probands developing hearing loss earlier. ${ }^{39}$

The probabilities of clinical events in the model are shown in Table 1. We used the US mutation prevalence estimate from 
Table 1

Model parameters used in analysis

\begin{tabular}{|c|c|c|c|}
\hline Parameters & Base-case & Range of estimates for sensitivity analysis & Source \\
\hline \multicolumn{4}{|l|}{ Probabilities } \\
\hline \multicolumn{4}{|l|}{ All patients } \\
\hline Variant prevalence (per 10,000) & 8.6 & $0.5-48.5$ & Refs. $14-16$ \\
\hline Lifetime exposure to aminoglycosides (AG) & 0.50 & $0.30-0.80$ & Refs. 30,31 \\
\hline Sensitivity of A1555G test & 0.999 & $0.949-0.999$ & Athena Diagnostics, Inc. \\
\hline Specificity of A1555G test & 0.870 & $0.830-0.930$ & Athena Diagnostics, Inc. \\
\hline $\begin{array}{l}\text { Increase in absolute risk of death from Pseudomonas } \\
\text { infection due to use of second-line therapy }\end{array}$ & 0.000 & $0.000-0.028$ & Ref. 29 \\
\hline \multicolumn{4}{|l|}{ Patients without variant } \\
\hline Mild or moderate hearing loss attributable to AG & 0.060 & $0.03-0.09$ & Ref. 6 \\
\hline Severe hearing loss attributable to AG & 0.012 & $0.006-0.018$ & Ref. 8 \\
\hline Late-onset hearing loss attributable to AG & 0.01 & $0.005-0.015$ & Ref. 6 \\
\hline Late-onset hearing loss given no exposure & 0.002 & $0.001-0.003$ & Ref. 41 \\
\hline \multicolumn{4}{|l|}{ Patients with variant } \\
\hline Mild or moderate hearing loss attributable to AG & 0.66 & $0.56-0.76$ & Ref. 42 \\
\hline Severe hearing loss attributable to AG & 0.34 & $0.34-1.00$ & Ref. 42 \\
\hline Late-onset hearing loss in exposed and unexposed & 0.40 & $0.30-0.60$ & Ref. 40,43 \\
\hline \multicolumn{4}{|l|}{ Utilities (quality of life) } \\
\hline CF patient without hearing loss & 0.80 & $0.75-0.85$ & Ref. 44 \\
\hline Patient with cochlear implant & 0.80 & $0.75-0.85$ & Ref. 45 \\
\hline CF patient with mild hearing loss & 0.91 & $0.85-0.95$ & Ref. 46 \\
\hline \multicolumn{4}{|l|}{ Costs (US\$2006) } \\
\hline Cost of A1555G test & $\$ 345$ & $\$ 259-431$ & Athena Diagnostics, Inc. \\
\hline Cost of cochlear implant & $\$ 81,000$ & $\$ 60,750-101,250$ & Ref. 47,48 \\
\hline Cost of annual maintenance of cochlear implant & $\$ 2,170$ & $\$ 1,600-2,700$ & Ref. 47,48 \\
\hline Tobramycin $375 \mathrm{mg}$ IV every $24 \mathrm{hr}$ for 14 days & $\$ 505$ & $\$ 379-631$ & CHRMC \\
\hline Cost of mild or moderate hearing loss & $\$ 680$ & $\$ 510-850$ & Ref. 35 \\
\hline Ceftazidime $2000 \mathrm{mg}$ IV every $8 \mathrm{hr}$ for 14 days & $\$ 1,300$ & $\$ 975-1,625$ & CHRMC \\
\hline Ciprofloxacin $500 \mathrm{mg}$ IV every $8 \mathrm{hr}$ for 14 days & $\$ 2,600$ & $\$ 1,950-3,250$ & CHRMC \\
\hline
\end{tabular}

CHRMC, Children's Hospital and Regional Medical Center, Seattle, WA.

the Tang et al. study as the base-case value for the probability of a CF patient testing positive for the mutation (8.6 per $10,000) .{ }^{14}$ Two studies investigating the prevalence of the variant in different populations worldwide have estimated the prevalence to be between 0.47 and 48.5 per $10,000.15,16$ The sensitivity and specificity of the A1555G test were reported to be $99.9 \%$ and $87.0 \%$, respectively (personal communications with Athena Diagnostics Inc., November 16, 2006).

Probabilities for each of the clinical outcomes were calculated depending on the combination of exposure and variant status. One factor that must be accounted for when examining mitochondrial mutations is whether the variant is homoplasmic (variant is found uniformly in the mitochondrial genome in all cells) or heteroplasmic (cells contain a mix of mitochon- dria, some with and some without the variant) as this affects penetrance. In the vast majority of studies, the $\mathrm{A} 1555 \mathrm{G}$ variant is reported as homoplasmic, but in two families the variant was reported as heteroplasmic. ${ }^{15,49,50}$ However, there seems to be variability in the clinical expression of the variant even when it is homoplasmic because variant-positive individuals manifest mild to severe or profound hearing loss in the presence of aminoglycoside exposure. As explained below, this variability is accounted for in the range of probabilities included for each of the potential clinical outcomes.

For variant-positive individuals, the probability of mild hearing loss as a result of aminoglycoside exposure was estimated at $66 \%$. This was based on one study by Usami et al. that categorized the degree of hearing loss. ${ }^{42}$ All other published 
studies examined individuals or families identified by the presence of severe to profound hearing loss. Therefore these studies estimate that $100 \%$ of individuals with the mutation who are exposed develop severe to profound hearing loss. We used $34 \%$ and $66 \%$ as the base-case analysis for severe or profound and mild or moderate aminoglycoside hearing loss, respectively. We varied these estimates to assume $100 \%$ of individuals experienced severe or profound hearing loss in a sensitivity analysis. The probability of developing late-onset hearing loss was estimated to be $40 \%$ in all individuals with the mutation, whether or not they were exposed to aminoglycoside. This figure was estimated from a study of 70 Spanish families with sensorineural hearing loss. ${ }^{40}$ The range of estimates used in the sensitivity analysis was estimated from several other familial studies and are shown in Table 1.

The probability of developing mild hearing loss as a result of aminoglycoside exposure has been reported to be between $0 \%$ and $16 \%$ in the general population. ${ }^{6}$ For the base-case analysis we used the median of these studies, approximately $7.5 \%{ }^{6}$ However, approximately $15 \%$ of all aminoglycoside-induced hearing loss is thought to be attributable to this mutation. ${ }^{9}$ Therefore, this amount was subtracted from the populationbased estimate to account for the fact that this estimate is likely to include people with the mutation. Although the majority of these studies do not describe whether hearing loss was mild, moderate, severe, or profound, one study estimated that approximately $2-4 \%$ of hearing loss resulting from aminoglycoside exposure was clinically significant. ${ }^{8}$ The probability of developing hearing loss by age 35 was estimated to be approximately $0.2 \%$ based on estimates from the National Health Information Survey data collected between 1997 and 2003 for adults between the ages of $18-44{ }^{41}$

\section{Health state utilities}

The effectiveness measure used in this analysis was QALYs. QALYs for each type of hearing outcome (i.e., mild, severe, none) were calculated by multiplying the length of time spent in each state by the quality of life associated with each state. The quality of life associated with a specific type of hearing loss is defined by a health utility score and ranges from 0 (death) to 1 (perfect health). Health state utilities associated with each type of hearing outcome and with CF are reported in Table 1. To determine the utility associated with an individual with CF, a literature review was conducted on CF patients. Health utility scores came from a sample of patients, doctors, and parents of individuals with CF. Patients estimated their quality of life to be approximately 0.9 using the time-tradeoff methodology whereas parents estimated the quality of life for CF patients to be around 0.7. ${ }^{44}$ The average value (0.8) was used in the basecase. A meta-analysis conducted on hearing loss for people with cochlear implants was used to derive utilities for individuals living with cochlear implants. Although quality of life changes depending on time with cochlear implants (with lower quality of life scores reported 1 year after implant, and higher scores for 2-3 years with implant), an average utility score was used for this analysis. After pooling seven studies assessing the health utility (utilizing several different instruments to measure quality of life) of adults with cochlear implants, Cheng and Niparko calculated an average utility of 0.80 .48 The health utility score for individuals with mild hearing loss (no cochlear implant) was estimated to be 0.91 based on a study assessing quality of life in individuals with less-severe sensorineural hearing. ${ }^{46}$ Although this study was not specific to aminoglycoside-induced hearing loss, it was the only estimate found in the literature that assessed quality of life for individuals with "mild" hearing loss. To obtain an overall health state utility, utility scores associated with each type of hearing loss were multiplied by utility scores associated with CF. All QALYs were discounted at $3 \%$ per year.

\section{Costs}

Costs utilized in this model were estimated from published cost-effectiveness reports on cochlear implantation, economic analyses of societal costs of hearing loss, test manufacturer, and data from Children's Hospital and Regional Medical Center in Seattle, WA. All costs were discounted at 3\% per year for the base-case analysis. Costs include direct costs associated with the cochlear implant procedure as well as the cost of the device and costs associated with rehabilitation, maintenance, followup, and drug costs. All costs are provided in Table 2 and are updated to 2006 dollars using the Consumer Price Index for medical care and medical devices. Most indirect and direct medical costs associated with the cochlear implant procedure were estimated from a 1999 cost-utility study of the multichannel cochlear implant in adults. ${ }^{47}$ Costs of long-term medical follow-up for patients were gathered from another economic study of cochlear implants. ${ }^{48}$

Costs associated with mild or moderate hearing loss were obtained from the Hearing Review 2006 Dispenser Survey. ${ }^{35}$ We averaged costs for all reported hearing aid types and the

Table 2

Results of cost-effectiveness analysis

\begin{tabular}{|c|c|c|c|c|}
\hline & $\begin{array}{l}\text { Probability of aminoglycoside-induced } \\
\text { severe hearing loss }\end{array}$ & $\begin{array}{c}\text { Total } \\
\text { cost (US\$2006) }\end{array}$ & $\begin{array}{c}\text { Quality-adjusted } \\
\text { life-years (QALYs) }\end{array}$ & $\begin{array}{l}\text { Incremental cost- } \\
\text { effectiveness ratio }^{a}\end{array}$ \\
\hline A1555G test & 0.0066 & $\$ 1603$ & 17.16467 & \\
\hline No A1555G test & 0.0078 & $\$ 1265$ & 17.16041 & \\
\hline Difference & -0.0012 & $\$ 338$ & 0.00426 & $\$ 79,300 / \mathrm{QALY}$ \\
\hline
\end{tabular}

${ }^{a}$ Discounted at 3\% per year. 
type of health care professional dispensing them and used this as our cost estimate for mild or moderate hearing loss. This is an estimate of hearing aid cost as well as services such as fitting that are bundled into the initial cost of the device. Because an average of $35 \%$ of individuals with mild or moderate hearing loss use hearing aids, we multiplied our final cost estimate by 0.35 .

Antibiotic costs were calculated using data from Children's Hospital and Regional Medical Center in Seattle, WA. The cost of the genetic test was obtained from the test manufacturer (personal communications with representative of Athena Diagnostics Inc., November 16, 2006).

\section{Sensitivity analyses}

To explore uncertainty in the model related to assumptions and uncertainty in the parameter estimates, one-way sensitivity analyses were conducted. Model parameters were individually varied over the ranges shown in Table 1 , while holding all other model estimates constant. The ranges for clinical and utility estimates were based on the ranges of values reported in the literature when possible, and cost estimates were varied by $\pm 25 \%$ of the base-case values. To explore the impact of improved test specificity, in a scenario analysis we modeled the inclusion of validation of positive test results, at an additional cost of $\$ 690$ (double the initial test cost), with an improvement in overall specificity from $87.0 \%$ to $99.9 \%$.

\section{RESULTS}

In the base-case analysis, the testing strategy led to a decrease in the incidence of severe aminoglycoside-induced hearing loss of $0.12 \%$ and an increase in QALYs of 0.00426 (1.6 days) (Table 2). The average discounted cost for the testing strategy was approximately $\$ 338$ more than the no test strategy. The ICER was thus $\$ 79,300$ per QALY gained.

However, these results were highly variable. In sensitivity analyses, testing resulted in cost per QALY gained ranging from $\$ 33,000$ to testing being dominated by the no testing strategy (higher costs and lower QALYs) (Table 3). The results were most sensitive to uncertainty in the estimate of mortality attributable to avoiding aminoglycoside use. In a threshold analysis, an increase of $0.8 \%$ in the absolute risk of mortality by avoiding aminoglycoside led to equal QALYs for each strategy; at values higher than $0.8 \%$, testing resulted in a decrease in QALYs compared with no testing. The discount rate was also influential, as hearing loss and its associated costs were discounted more heavily in later years. Other important parameters, as expected, were the probability of being exposed to an aminoglycoside, test specificity and cost, utility of hearing loss, and mutation prevalence. The risk of aminoglycoside-induced hearing loss (both mild or moderate and severe) in patients without the mutation was also a driving factor in the analysis. Although this may seem counterintuitive at first, this is a result of the high percentage $(>99.5 \%)$ of false-positives (i.e., true mutation-negative) patients who are switched to ciprofloxa- cin, thus avoiding the risk of aminoglycoside-induced hearing loss.

In scenario analysis with positive test result validation and improved specificity, significantly fewer patients tested positive $(1.1 \%$ vs. $13.1 \%)$ and were targeted for ciprofloxicin rather than aminoglycoside therapy. Interestingly, because more patients received aminoglycosides in this scenario (regardless of genetic status), the improvement in QALYs was actually less than in the base-case (0.001 vs. 0.004$)$, and the ICER was significantly larger, $\$ 670,000 / \mathrm{QALY}$. When the potential increase in mortality associated with using ciprofloxacin is included, testing resulted in a 0.001 decrease in QALYs compared with no testing.

\section{DISCUSSION}

We estimated the incremental cost-effectiveness of screening for the A1555G variant compared with not screening (standard of care) in a hypothetical cohort of patients with CF. In our base-case analysis, we found that the testing strategy slightly decreased the risk of severe aminoglycoside-induced hearing loss and slightly increased QALYs. However, these small improvements in patient outcomes do not seem to be cost-effective, with an ICER of $\$ 79,300$ per QALY gained, compared with the commonly cited (although approximate) threshold of $\$ 50,000$ per QALY in the United States. As importantly, there was significant uncertainty in our results primarily because of the paucity of data; indeed, it is possible that patients, on average, could experience worse outcomes as a result of the avoidance of first-line antibiotic therapy. In addition, our results suggest that improvements in the specificity of the test would not likely lead to an effective or cost-effective strategy because of the relatively small average gains in qualityadjusted life expectancy, the potential risk of poorer response to alternative antibiotics, and in particular, the low prevalence of the mutation.

Our systematic review of studies of the A1555G mutation revealed few data, with much of the available data of relatively poor quality. Most studies were small ( $<50$ people) and conducted on either large high-risk pedigrees of maternally inherited hearing loss or conducted on individuals who already had severe to profound hearing loss. Despite this lack of data, the test is clinically available and being marketed directly to clinicians.

The clinical and economic implications of our findings may be important. The results are driven by one key factor: the low prevalence of the mutation (approximately 8.5 per 10,000). Because of the low prevalence, approximately 1200 patients would have to be screened at a cost of $\$ 338,000$ to identify a single patient with a mutation. Given that there are approximately 60,000 patients worldwide with CF, only about 50 patients would be expected to have the mutation. Furthermore, because the test has less-than-perfect specificity $(87 \%)$, over $95 \%$ of patients that test positive will actually not have the mutation. Thus, if clinicians switch therapy based on the test result, they will be doing so unnecessarily in the great majority 
Table 3

Results of sensitivity analyses

\begin{tabular}{|c|c|c|c|c|}
\hline Variable & Low input & High input & Low result & High result \\
\hline Absolute risk of mortality from aminoglycoside avoidance & 0 & 0.028 & $\$ 79,000$ & QALYs decreased \\
\hline Discount rate & $0 \%$ & $5 \%$ & $\$ 33,000$ & $\$ 134,000$ \\
\hline $\begin{array}{l}\text { Probability of being exposed to aminoglycosides in a CF patient } \\
\text { population (lifetime) }\end{array}$ & 0.3 & 0.8 & $\$ 49,000$ & $\$ 133,000$ \\
\hline Specificity of A1555G test & 0.83 & 0.93 & $\$ 62,000$ & $\$ 138,000$ \\
\hline Probability of mild or moderate hearing loss in amino-exposed A1555G(-) & 0.03 & 0.09 & $\$ 61,000$ & $\$ 118,000$ \\
\hline Utility for mild hearing loss & 0.85 & 0.95 & $\$ 60,000$ & $\$ 117,000$ \\
\hline Cost of test & $\$ 259$ & $\$ 431$ & $\$ 59,000$ & $\$ 100,000$ \\
\hline Mutation prevalence & 0.00005 & 0.00485 & $\$ 53,000$ & $\$ 87,000$ \\
\hline Probability of amino-induced severe hearing loss in amino-exposed A1555G(-) & 0.006 & 0.018 & $\$ 66,000$ & $\$ 97,000$ \\
\hline Utility of severe hearing loss (cochlear implant) & 0.75 & 0.85 & $\$ 73,000$ & $\$ 87,000$ \\
\hline Cost of ciprofloxacin & $\$ 1,950$ & $\$ 3,250$ & $\$ 73,000$ & $\$ 85,000$ \\
\hline $\begin{array}{l}\text { Probability of late-onset hearing loss in amino-exposed A1555G(-) with no } \\
\text { amino-induced hearing loss }\end{array}$ & 0.005 & 0.015 & $\$ 74,000$ & $\$ 85,000$ \\
\hline Utility for CF & 0.75 & 0.85 & $\$ 75,000$ & $\$ 85,000$ \\
\hline Cost of cochlear implant yr 1 & $\$ 60,750$ & $\$ 101,250$ & $\$ 76,000$ & $\$ 83,000$ \\
\hline Probability of amino-induced severe hearing loss in amino-exposed A1555G(+) & 0.34 & 1.00 & $\$ 73,000$ & $\$ 79,000$ \\
\hline Cost maintenance cochlear implant yrs $2+$ & $\$ 1,600$ & $\$ 2,700$ & $\$ 78,000$ & $\$ 81,000$ \\
\hline Probability of late-onset hearing loss in A1555G(-) with no amino exposure & 0.001 & 0.003 & $\$ 78,000$ & $\$ 81,000$ \\
\hline Probability of late-onset hearing loss in A1555G(+) with no amino exposure & 0.3 & 0.6 & $\$ 79,000$ & $\$ 81,000$ \\
\hline Cost of tobramycin & $\$ 379$ & $\$ 631$ & $\$ 78,000$ & $\$ 81,000$ \\
\hline Probability of late-onset hearing loss in amino-exposed and A1555G(-) & 0.03 & 0.07 & $\$ 78,000$ & $\$ 81,000$ \\
\hline Probability of late-onset hearing loss in amino-exposed A1555G(+) & 0.3 & 0.6 & $\$ 79,000$ & $\$ 80,000$ \\
\hline Sensitivity of A1555G test & 0.949 & 0.999 & $\$ 79,000$ & $\$ 80,000$ \\
\hline Cost of mild hearing loss & $\$ 510$ & $\$ 850$ & $\$ 79,000$ & $\$ 80,000$ \\
\hline Cost of ceftazidime & $\$ 975$ & $\$ 1,625$ & $\$ 79,000$ & $\$ 79,000$ \\
\hline Probability of mild or moderate hearing loss in amino-exposed and A1555G(+) & 0.56 & 0.76 & $\$ 79,000$ & $\$ 79,000$ \\
\hline
\end{tabular}

Note that the low and high inputs for each parameter do not necessarily correspond with the low and high results, respectively.

of cases. Clinicians should interpret the results of A1555G testing with appropriate caution, payers should critically evaluate reimbursement policies, and from an opportunity cost perspective, both clinicians and payers should consider alternative uses of health care resources and the benefits they might bring to their patients.

There are limitations in our analysis. First, estimates of variant prevalence are based on a paucity of data. The Tang et al. study represents the only large population-based study of A1555G prevalence, and this estimate is based on the existence of one bloodspot. ${ }^{14}$ Therefore, the confidence interval surrounding this estimate is large and as shown in our sensitivity analysis, fluctuations in variant prevalence result in significant changes in the ICER. Second, the majority of studies of the association between genotype and the aminoglycoside-induced hearing loss were conducted in families with a high prevalence of hearing loss. These families may represent a high-risk subgroup of all people with the variant. Thus, the estimated $100 \%$ penetrance of the mutation after exposure may be an overestimation. Similarly, the $39.9 \%$ penetrance without exposure may be an overestimation and thus model results may be reflective of the situation in carriers from highrisk families, which is likely to represent a small proportion of all carriers. Third, most investigations of aminoglycoside-induced hearing loss did not characterize the degree of hearing loss as a result of ototoxic effects. Many of the ototoxic effects of aminoglycosides in the general population are reported as subclinical or mild (though most studies have short follow-up periods). Because ototoxic effects of this drug are thought to be progressive, there may be additional unreported cases of lateonset hearing loss in a cohort of exposed individuals. Fourth, there may be some low risk of hearing loss over years of chronic exposure to inhaled tobramycin, but there is a paucity of longterm data and it would be difficult to dissect the effects of 
inhaled versus intermittent intravenous tobramycin on hearing loss in this population. Fifth, it has been reported that specific clinical characteristics of CF patients may provide a degree of protection to the ototoxic effects of aminoglycosides. ${ }^{6}$ However, this population is exposed to higher doses more often than most people exposed to aminoglycosides in routine clinical practice. Therefore, we assumed that the protective effect may make them equivalent to other populations of patients exposed to smaller doses for shorter periods of time. Lastly, although CF patients are likely to be exposed to aminoglycosides, the high mortality associated with Pseudomonas respiratory infections and the lower effectiveness of alternative therapies may be particularly challenging in this population and mitigate against the benefits of the A1555G test.

In conclusion, there are limited data on the A1555G mitochondrial mutation, although it is likely a significant risk factor for aminoglycoside-induced hearing loss. However, because of the low prevalence of the mutation, the associated high falsepositive rate, and the potential harm associated with a falsepositive screen in the CF population, routine testing does not seem to be cost-effective or clinically warranted in CF patients given currently available data.

\section{ACKNOWLEDGMENTS}

This work was supported by the Maternal and Child Health Bureau, Health Resources and Services Administration Genetic Services Policy U35MC02601 and U35MC02602, and the Center for Genomics and Health care Equality Grant P50 HG003374 from the US National Institutes of Health.

\section{References}

1. Magnet S, Blanchard J. Molecular insights into aminoglycoside action and resistance. Chem Rev 2005;105:477-498.

2. Forge A, Schacht J. Aminoglycoside antibiotics. Audiol Neurootol 2000;5:3-22.

3. McCracken GH Jr. Aminoglycoside toxicity in infants and children. Am J Med 1986;80:172-178

4. Nakashima T, Teranishi M, Hibi T, Kobayashi M. Vestibular and cochlear toxicity of aminoglycosides: a review. Acta Otolaryngol 2000;120:904-911.

5. Munckhof W, Grayson M, Turnidge J. A meta-analysis of studies on the safety and efficacy of aminoglycosides given either once daily or as divided doses. J Antimicrob Chemother 1996;37:645-663.

6. Mulheran M, Degg C, Burr S, Morgan D, et al. Occurrence and risk of cochleotoxicity in cystic fibrosis patients receiving repeated high-dose aminoglycoside therapy. Antimicrob Agents Chemother 2001;45:2502-2509.

7. Gilbert D. Aminoglycosides. In: Mandell G, Bennett J, Dolin R, editors. Mandell, Douglas, and Bennett's principles and practices of infectious diseases, 5th ed. New York: Churchill Livingstone, 2000:307-336.

8. Periti P. Pharmacoeconomic evaluation of once-daily aminoglycoside treatment. J Chemother 1995;7:380-394.

9. Fischel-Ghodsian N, Prezant TR, Chaltraw WE, Wendt KA, et al. Mitochondrial gene mutation is a significant predisposing factor in aminoglycoside ototoxicity. Am J Otolaryngol 1997;18:173-178.

10. Li Z, Li R, Chen J. Mutational analysis of the mitochondrial 12S rRNA gene in Chinese pediatric subjects with aminoglycoside-induced and non-syndromic hearing loss. Hum Genet 2005;117:9-15.

11. Zhao H, Li R, Wang Q, Yan Q, et al. Maternally inherited aminoglycoside-induced and nonsyndromic deafness is associated with the novel C1494T mutation in the mitochondrial 12 S rRNA gene in a large Chinese family. Am J Hum Genet 2004;74:139-152.

12. Jaber J, Shohat M, Bu X, Fischel-Ghodsian N, et al. Sensorineural deafness inherited at a tissue specific mitochondrial mutation. J Med Genet 1992;29:86-90.

13. Nance W. The genetics of deafness. Mental Retard Dev Disabil Res Rev 2003;9:102-109.

14. Tang HY, Hutcheson E, Neill S, Drummond-Borg M, et al. Genetic susceptibility to aminoglycoside ototoxicity: how many are at risk? Genet Med 2002;4: $336-345$.
15. Fischel-Ghodsian N. Mitochondrial deafness. Ear Hearing 2003;24:313

16. Lehtonen M, Uimonen S, Hassinen I, Majamaa K. Frequency of mitochondrial DNA point mutations among patients with familial sensorineural hearing impairment. EurJ Pediatr 2000;8:315-319.

17. Tekin M, Duman T, Bogoclu G, Incesulu A, et al. Frequency of mtDNA A1555G and A7445G mutations among children with prelingual deafness in Turkey. Eur J Pediatr 2003; $162: 154-158$

18. Torroni A, Cruciani F, Rengo C, Sellitto D, et al. The A1555G mutation in the $12 \mathrm{~S}$ rRNA gene of human mtDNA: recurrent origins and founder events in families affected by sensorineural deafness. Am J Hum Genet 1999;65:1349-1358.

19. Prezant T, Agapian J, Bohlman M, Bu X, et al. Mitochondrial ribosomal RNA mutation associated with both antibiotic-induced and non-syndromic deafness. Nat Genet 1993;4:289-294

20. Kupka S, Toth T, Wrobel M, Zeissler U, et al. Mutation A1555G in the 12S rRNA gene and its epidemiological importance in German, Hungarian, and Polish patients. Hum Mutat 2002;19:308-309.

21. del Castillo FJ, Rodriguez-Ballesteros M, Martin Y, Arellano B, et al. Heteroplasmy for the $1555 \mathrm{~A}>\mathrm{G}$ mutation in the mitochondrial $12 \mathrm{~S}$ rRNA gene in six Spanish families with non-syndromic hearing loss. J Med Genet 2003;40:632-636.

22. Pandya A. Nonsyndromic hearing loss, mitochondrial: National Institute of Health. Seattle, WA: University of Washington, 2004. Available at: http://www.genereviews. org.

23. Athena Diagnostics Inc Website. Available at: http://www.athenadiagnostics.com/ content/index.jsp. Accessed December 5, 2005.

24. Foundation CF. Patient Registry 2004, Annual Report Bethesda, MD: Cystic Fibrosis Foundation; 2004.

25. Gibson RL, Burns JL, Ramsey BW. Pathophysiology and management of pulmonary infections in cystic fibrosis. Am J Respir Crit Care Med 2003;168:918-951.

26. Foundation CF. Patient registry 2005, annual report. Bethesda, MD: Cystic Fibrosis Foundation, 2005.

27. TreeAge Pro 2005 Suite [computer program]. Willamstown, MA: TreeAgePro. 2005.

28. Ramsey B. Management of pulmonary disease in patients with cystic fibrosis. NEngl J Med 1996;335:179-187.

29. Shawar R, MacLeod D, Gerber R, Burns JL, et al. Activities of tobramycin and six other antibiotics against Pseudomonas aeruginosa isolates from patients with cystic fibrosis. Antimicrob Agents Chemother 1999;43:2877-2880.

30. Foundation CF. Cystic fibrosis foundation registry. Bethesda, MD: Cystic Fibrosi Foundation, 2003.

31. Hoiby N. Prospects for the prevention and control of pseudomonal infection in children with cystic fibrosis. Paediatr Drugs 2000;2:451-463.

32. Tono T, Kiyomizu K, Matsuda K, Komune S, et al. Different clinical characteristic of aminoglycoside induced profound deafness with and without the $1555 \mathrm{~A}-\mathrm{G}$ mitochondrial mutation. J Otorhinolaryngol Relat Spec 2001;63:25-30.

33. Tono T, Ushisako Y, Kiyomizu K, Usami S, et al. Cochlear implantation in a patient with profound hearing loss with the A1555G mitochondrial mutation. Am J Otol 1998;19:754-757.

34. Cortopassi G, Hutchin T. A molecular and cellular hypothesis for aminoglycosideinduced deafness. Hear Res 1994;78:27-30.

35. Strom K. The HR. 2006 Dispenser Survey. Hear Rev 2006;13:16-39.

36. Church D, Kanga J, Kuhn R, Rubio TT, et al. Sequential ciprofloxacin therapy in pediatric cystic fibrosis: comparative study vs. ceftazidime/tobramycin in the treatment of acute pulmonary exacerbations. Pediatr Infect Dis J 1997;16: 97-105.

37. Mayer-Hamblett N, Rosenfeld M, Emerson J, Goss CH, et al. Developing cystic fibrosis lung transplant referral criteria using predictors of 2-year mortality. Am J Respir Crit Care Med 2002;166(12 pt 1):1550-1555.

38. Emerson J, Rosenfeld M, McNamara S, Ramsey B, et al. Pseudomonas aeruginosa and other predictors of mortality and morbidity in young children with cystic fibrosis. Pediatr Pulmonol 2002;34:91-100.

39. Matsunaga T, Kumanomido H, Shiroma M, Ohtsuka A, et al. Deafness due to A1555G mitochondrial mutation without use of aminoglycoside. Laryngoscope 2004;114:1085-1091.

40. Estivill X, Govea N, Barcelo E, Perelló E, et al. Familial progressive sensorineural deafness is mainly due to the mtDNA A1555G mutation and is enhanced by treatment of aminoglycosides. Am J Hum Genet 1998;62:27-35.

41. Mitchell RE. Summary of estimates for the size of the deaf population in the USA. Washington DC: Gallaudet Research Institute, 2005.

42. Usami S-I, Abe S, Akita J, Namba A, et al. Prevalence of mitochondrial gene mutations among hearing impaired patients. J Med Genet 2000;37:38-40.

43. Hutchin T, Cortopassi G. Mitochondrial defects and hearing loss. Cell Mol Life Sci 2000;57:1927-1937.

44. Rowley P, Loader S, Kaplan R. Prenatal screening for cystic fibrosis carriers: an economic evaluation. Am J Hum Genet 1998;63:1160-1174. 


\section{Veenstra et al.}

45. Cheng A, Niparko J. Cost-utility of the cochlear implant in adults. Arch Otolaryngol Head Neck Surg 1999;125:1214-1218.

46. McIntyre P, Hall J, Leeder S. An economic analysis of alternatives for childhood immunisation against Haemophilus influenza type B disease. Australian J Public Health 1994;18:394-400.

47. Palmer C, Niparko J, Wyatt R, Rothman M, et al. A prospective study of the cost-utility of the multichannel cochlear implant. Arch Otolaryngol Head Neck Surg 1999;125: $1221-1228$.
48. Carter R. Economic evaluation of the cochlear implant. Australia: Centre for Health Program Evaluation, 1994.

49. el Schahawi M, Lopez DM, Sarrazin AM, Shanske AL, et al. Two large Spanish pedigrees with nonsyndromic sensorineural deafness and the mtDNA mutation at 1555 in the 12s rRNA gene: evidence of heteroplasmy. Neurology 1997;48:453-456.

50. Morales AC, Gallo JT, Castillo ID, Moreno MP, et al. Audiometric features of familial hearing impairment transmitted by mitochondrial inheritance (A1555G). Acta Otorrinolaringol Esp 2002;53:641-648. 